\title{
PROBLEME VON DIREKTINVESTITIONEN IN ENTWICKLUNGSLANDERN UND MÖGLICHKEITEN IHRER RECHTLICHEN ABSICHERUNG
}

\author{
Von Karl Fasbender und Karl Wolfgang Menck
}

\section{Volumen und Struktur der Direktinvestitionen}

Direktinvestitionen ${ }^{1}$ in den Staaten der Dritten Welt gehören zu den am meisten geförderten und zugleich $\mathrm{zu}$ den umstrittensten Instrumenten der Entwicklungspolitik. Gefördert, weil sie als eine ideale Kombination zur Vermittlung von Kapital und technischem know-how gelten; umstritten, weil auf ihnen der Vorwurf des Neokolonialismus lastet. Die Bereitstellung von Entwicklungskapital durch ausländische Investoren gewinnt ständig an Bedeutung. Allein 1969 investierten die Unternehmer der DAC-Staaten für 2,57 Mrd. US-Dollar². Da dieses Kapital nicht wie die staatlichen und privaten Kredite zu einem bestimmten Satz verzinst und nicht innerhalb eines bestimmten Zeitraumes zurückgezahlt werden muß, trägt es dazu bei, das Verschuldungsproblem dieser Länder zu mildern. Die anfallenden Gewinne werden, sofern sie nicht reinvestiert werden, in der Regel erst nach einer längeren Anlaufzeit transferiert. Zusätzliche Effekte für die Entlastung der Handelsbilanz ergeben sich aus der Substitution von Importgütern und der möglichen Erhöhung der Exporterlöse. Dagegen müssen allerdings die benötigten Zulieferungen aufgerechnet werden. Viele Entwicklungsländer fördern bzw. dulden daher nur solche Investitionen, die zumindest mittel- und langfristig ihren Bedarf im Inland decken können.

Besonders günstig erweisen sich die Direktinvestitionen bei der Übermittlung von Technischem Wissen, das nur im Umgang mit Menschen und Geräten erworben werden kann - beispielsweise technische Fähigkeiten - und bei der Ausbildung des mittleren und gehobenen Managements. Da erfahrungsgemäß ein Teil der Fachkräfte in andere Tätigkeitsbereiche abwandert und eigene Werkstätten gründet, erhält das betreffende Land einen Versorgungsdienst und das Unternehmen ein Netz von Reparaturbetrieben ${ }^{3}$. Nicht zuletzt schaffen die ausländischen Privatinvestitionen neue Arbeitsplätze. So sind beispielsweise in 50,9\% der deutschen Auslandsunternehmen in der Dritten Welt mehr als 500 Arbeitskräfte beschäftigt; bei $26,8 \%$ sind mehr als 2000 Arbeitnehmer tätig4. Dieser Beschäftigungseffekt wird durch die von den Direktinvestitionen induzierten inländischen Investitionen verstärkt.

1 Direktinvestitionen bzw. private Auslandsinvestitionen sind langfristige Kapitalexporte mit Beteiligungscharakter.

2 Vgl. OECD, Development Assistance, 1970 Review, Tabelle 6, S. 176 f.

3 Vgl. D. Kebschull, unter Mitarbeit von K. Fasbender und A. Naini, Entwicklungspolitik - eine Einführung, Düsseldorf 1971.

4 Auswertung einer Umfrage des HWWA-Institut für Wirtschaftsforschung Hamburg. Befragt wurden 92 deutsche Unternehmen in 25 Ländern. Vgl. Entwicklungspolitik, a. a. O., und D. Kebschull, K. W. Menck, Deutsche Direktinvestitionen in der Dritten Welt - Motive und Probleme, Veröffentlichung in Vorbereitung. Zur Veröffentlichung der Vorergebnisse siehe: A. Al-Ani: German Investment in Developing Countries, Intereconomics, Nr. 7, 1969, S. $219 \mathrm{ff}$. 
Die Kritiker dieser Investitionen wenden vor allem ein, daß die ausländischen Unternehmungen einen bedeutenden wirtschaftlichen Machtfaktor darstellen und so einen unerwünschten politischen Einfluß ausüben können. Hierfür lassen sich in der Tat viele - meist amerikanische - Beispiele anführen. Obwohl diese auch noch keine Verallgemeinerungen zulassen, bilden sie in der politischen Diskussion doch ein nicht $\mathrm{zu}$ unterschätzendes Argument. Kritisiert wird ferner, daß die Investoren überhöhte Gewinne erzielen und transferieren ${ }^{5}$. Auch hier beschränkt man sich auf einzelne Beispiele. Nach einer im Auftrage des Bundesministeriums für wirtschaftliche Zusammenarbeit erstellten Studie erzielten die deutschen Unternehmer in den Jahren 1965 bis 1967 eine durchschnittliche Netto-Rendite von 6,25\%. Die amerikanischen und englischen Investoren erwirtschafteten 1967 dagegen eine Rendite von etwa $12 \%$ bzw. 9\%. Die Reinvestitionsquote betrug bei den deutschen Investitionen $70 \%$, bei den englischen (ohne Olindustrie), ca. $34 \%$ und bei den amerikanischen etwa $18 \%{ }^{6}$.

Damit sind die Gewinne niedriger als allgemein angenommen. Die Feststellung, daß die Erträge der deutschen Direktinvestitionen am niedrigsten sind und damit den Vorstellungen der Entwicklungsländer am nächsten kommen, erklärt sich aus dem relativ jungen Datum dieser Auslandsunternehmungen. Die tatsächlichen Auswirkungen lassen sich jedoch nur an Hand der Zahlungsbilanz der einzelnen Entwicklungsländer feststellen.

Trotz aller Kritik wächst das Interesse der Entwicklungsländer an ausländischen Investitionen. Ursache ist die enge Verbindung von Entwicklungspolitik und dieser Form der privaten „Hilfe“. Darüber hinaus tragen gesetzliche Bestimmungen wie über den Transfer von Gewinnen und der in letzter Zeit in den Vordergrund tretende Typ von Partnerschaftsunternehmen mit inländischen Anteilseignern (Joint Ventures) dazu bei, die Kritik abzubauen. Dem steigenden Interesse in der Dritten Welt an privatem Auslandskapital steht eine Zunahme der Investitionen gegenüber. So wuchsen die deutschen Direktinvestitionen im Zeitraum von 1967 bis 1970 von 3,5 Mrd. DM auf 6,2 Mrd. DM. Ihr Anteil an den gesamten deutschen Auslandsinvestitionen stieg allerdings nur um 2,5\% auf $29 \% 0^{7}$. Sektorale Schwerpunkte stellen die Investitions-, Grundstoff- und Produktionsgüterindustrie dar. Auf sie entfielen von 1952 bis 1968 68,1\% der deutschen Investitionen ${ }^{8}$. Hieran zeigt sich, daß das ursprüngliche Motiv der Direktinvestitionen, die Sicherung von Rohstofflieferungen, an Bedeutung verloren hat. Deshalb trifft der Vorwurf nicht zu, deutsche Unternehmen beuteten die Rohstoffquellen der Dritten Welt aus. Nur 2,9\% der befragten Unternehmen hielten diesen Beweggrund für nennenswert.

5 Vgl. W. Kraus, Ausbeutung der Entwicklungsländer durch Privatinvestitionen? in: Materialien zur Entwicklungshilfekritik, Hrsg. Deutsche Stiftung für. Entwicklungsländer, Bonn-Lengsdorf, 1969, S. $35 \mathrm{ff}$.

6 Vgl. G. Grosche, R. Lehmann-Richter, Die Gewinne aus deutschen Direktinvestitionen in Entwicklungsländern, Düsseldorf 1970, S. $54 \mathrm{ff}$.

7 Angaben des BMWI in: Runderlasse Außenwirtschaft, in Bundesanzeiger seit 1967 (eigene Berechnungen).

8 Vgl. BMZ-Pressemitteilungen - Regional verteilen sich die Direktinvestitionen auf Lateinamerika $(60,9 \%)$, Europa $(17,3 \%)$, Afrika $(14,7 \%)$, und Asien $(7,1 \%)$. Berechnet nach: BMWI, Runderlaß Außenwirtschaft, Nr. $16 / 17$ vom 19. 4. 1970 im Bundesanzeiger, Jg. 22, Nr. 83 vom 6. 5. 1970. 


\section{Tabelle 1}

\section{Motive deutscher Investitionen in Entwicklungsländern}

Motive

Erschließung neuer Märkte

Zwangsmotive

(Patentsicherung, Importrestriktionen, Auftragssicherung)

Vergünstigungen steuerlicher und finanzieller Art seitens

a) der Bundesrepublik Deutschland

b) der Entwicklungsländer

Sicherung der Marktstellung davon wegen

\begin{tabular}{|c|c|}
\hline \multicolumn{2}{|c|}{ Antworten } \\
\hline bs. & in Prozent \\
\hline 9 & 24,8 \\
\hline 7 & 20,5 \\
\hline 6 & 12,9 \\
\hline 27 & 9,7 \\
\hline 9 & 3,2 \\
\hline & 12,6 \\
\hline
\end{tabular}

a) Exportrückganges 16

b) verschärften Wettbewerbs in der Bundesrepubl. Deutschland 7

c) Veränderungen in der Nachfragestruktur in Europa 6

d) sonstige Motive

Good-Will-Motive

Schaffung von zusätzlichem Absatz

für andere Unternehmensprodukte

Niedrige Löhne

Sicherung von Rohstofflieferungen

Niedrige Preise für Roh- und Werkstoffe

Mangel an Arbeitskräften

in der Bundesrepublik Deutschland

Sonstige Motive

Summe

\begin{tabular}{rrr} 
& 16 & 5,7 \\
& 7 & 2,5 \\
& 6 & 2,2 \\
24 & & \multicolumn{2}{c}{8,6} \\
& & \\
15 & & 5,4 \\
15 & & 5,4 \\
8 & & 2,9 \\
5 & & 1,8 \\
& & \\
3 & & 1,1 \\
11 & & 4,0 \\
278 & & 100,0
\end{tabular}

Anmerkung: Mehrfachbeantwortungen waren möglich.

Quelle: Umfrage des HWWA-Institut für Wirtschaftsforschung - Hamburg.

Die Investitionen tragen vielmehr dazu bei, die Produktions- und Exportstruktur zu diversifizieren. Angeführt wird die Motivskala von der Absicht, die Marktstellung zu sichern und neue Absatzmärkte zu erschließen $(37,4 \%$ ). Die in der Ơffentlichkeit am häufigsten genannten Motive, niedrige Preise für Produktionsfaktoren und Löhne, wurden von nur 7,2\% der Unternehmer für beachtenswert gehalten (vgl. Tabelle 1). Die Vorstellung, daß in diesen Staaten grundsätzlich billiger produziert wird, trifft tatsächlich nur bei $21,1 \%$ der befragten Unternehmungen zu. Dies erklärt sich zum großen Teil aus den unrealistischen Wechselkursen, die nicht das reale Austauschverhältnis wiedergeben. Bei $76 \%$ der deutschen Direktinvestitionen liegen die Gesamtkosten je Produktionseinheit über denen der in der Bundesrepublik getätigten Investitionen ${ }^{9}$.

Dazu kommen die Probleme, die sich aus der besonderen wirtschaftlichen, sozialen und politischen Situation im Anlageland ergeben. Hervorzuheben sind vor allem die bürokratischen Hemmnisse, die unzureichende Möglichkeit, notwendige Zulieferungen $\mathrm{zu}$ importieren, sowie die mangelnde Qualität der Arbeitskräfte (vgl. Tabelle 2). Hierdurch werden die Vorteile, die sich aus den Direktinvestitionen

9 Vgl. D. Kebschull, K. W. Menck, a. a. O. 
Tabelle 2

Probleme deutscher Direktinvestitionen in Entwicklungsländern

Problem

Bürokratische, administrative Hemmnisse im Anlageland

Einfuhrhemmnisse wegen Devisenmangels

Antworten gesamt

Mangelnde Qualität und irrationale Mentalität

der Arbeitskräfte

abs. in Prozent

$86 \quad 28,7$

$56 \quad 18,7$

Mangelnde Qualität der Roh- und Werkstoffe

und der Betriebsmittel

40

13,3

Lieferungsverzögerungen bei Ersatzteilen

sowie Roh- und Werkstoffen

Unzureichende Infrastruktur

$31 \quad 10,3$

Schwierigkeiten beim Gewinntransfer

25

8,3

Verständigungsschwierigkeiten

Unzureichende Marktgröße / politische Unstabilität

Keine Probleme

20

6,8

$1 \quad 0,3$

$1 \quad 0,3$

Summe

Anmerkung: Mehrfachbeantwortungen waren möglich.

Quelle: Umfrage des HWWA-Institut für Wirtschaftsforschung - Hamburg.

für die Unternehmer ergeben, zum Teil wettgemacht. Solange die Industrieländer im langfristigen Kapitalexport einen wesentlichen Teil ihrer Entwicklungshilfe sehen, müssen sie deshalb Maßnahmen zur Förderung der Direktinvestitionen ergreifen. Sie sollen vor allem die Furcht vor Enteignungen und anderen Eingriffen in das Eigentum der deutschen Unternehmen verhindern. Die Vorschläge der Weltbank, der OECD und der Vereinten Nationen sollen dieses Risiko für den Investor beseitigen, indem in den kapitalgebenden und kapitalnehmenden Ländern gleiche Rechte und Pflichten für das Eigentum an Produktionsmitteln vereinbart werden sollten. Dann würden unterschiedliche nationalstaatliche Rechtsvorschriften keine Rolle mehr spielen. Diesen Empfehlungen folgten bisher jedoch keine Taten. So müssen ersatzweise bilaterale Abkommen gerade mit Entwicklungsländern vereinbart werden, da dort die Begriffe von der Schutzwürdigkeit des Eigentums stärker von denen in Industrieländern abweichen.

\section{Bilaterale Investitionsförderungsverträge}

(Kapitalschutzabkommen)

Darin sehen die Investoren aber gerade das besondere Risiko, wenn sie Kapitalanlagen in Entwicklungsländern vornehmen. Deshalb müssen die Wünsche der Investoren nach größerer Rechtssicherheit mit den Vorstellungen der Entwicklungsländer in Kapitalschutzabkommen (Investitionsförderungsverträgen) abgestimmt werden. Sie regeln:

"- die Anerkennung materieller Verhaltensnormen in beiden Staaten

- die Sanktionen und 
- Vorkehrungen zur Schlichtung von Auseinandersetzungen."10

Damit sollen unterschiedliche Rechtsauffassungen in den Geber- und Nehmerländern aufeinander angeglichen werden. Dem Investor zeigen die Bestimmungen des Abkommens

- welche Eigentumsrechte im Anlageland eingehalten werden und

- wie er bei einer Verletzung und/oder Nichteinhaltung dieser Vereinbarungen seine Rechte wahren kann.

Diese Form der Rechtssicherheit ist die einzig mögliche Sicherung des Auslandskapitals. Weitergehende Sicherungen verlangen auch die Eigentümer nicht ${ }^{11}$. Sie wollen aber den Schutz des Völkerrechts für ihre Auslandsinvestitionen gewahrt wissen. „Der völkerrechtliche Begriff des Eigentums bietet nur einen Maximalrahmen, in den nach Völkerrecht Eigentumsrechte fallen können. Wieweit dieser Rahmen im Einzelfall ausgenutzt wird, hängt jeweils von der zuständigen nationalen Rechtsordnung $a b$, denn diese bestimmt, welche Arten von Eigentumsrechten sie entstehen lassen will. Diese sind dann völkerrechtlich geschützt ${ }^{12}$." Einmütigkeit besteht im Völkerrecht lediglich über die Entschädigungspflicht bei Enteignungen und enteignungsähnlichen Eingriffen ${ }^{13}$. Trotzdem erfolgten Enteignungen zum Teil ohne Entschädigung, zumal dann, wenn die Investoren den „Interessen " des Gastlandes zuwiderhandelten ${ }^{14}$. Schwierigkeiten bei der Bemessung der Entschädigung ergeben sich immer bei der Abgrenzung enteignungsähnlicher Tatbestände ${ }^{15}$. Zum Nutzen des Gastlandes und des Investors müssen dafür Wege des Ausgleichs gefunden werden. Angesichts der starken Verschuldung und ihrer negativen Handelsbilanzen wollen die Entwicklungsländer möglichst die aus Exporten und Entwicklungshilfeleistungen erhaltenen Devisen nicht für den Gewinntransfer ausländischer Investoren freigeben. Nur dann können sie ihre Schulden tilgen und die notwendigen industriellen Güter importieren. Jeder Gewinntransfer würde die dafür vorgesehenen Devisen mindern. Deshalb sollen nach Auffassung der Entwicklungsländer die Gewinne der Ausländischen Unternehmen im Entwicklungsland zur Kapitalbildung beitragen. Die Rechtsordnung der Industrieländer sieht demgegenüber in der Regel die freie Verfügung über die Gewinne vor. Setzen die Entwicklungsländer ihre Vorstellungen durch, werden unter Umständen entwicklungspolitisch notwendige Investitionen ausbleiben, da die Investoren nicht immer bereit sein werden, auf den Gewinntransfer zu verzichten ${ }^{\mathbf{1 6}}$.

Die Entwicklungsländer sind aber auf das ausländische Privatkapital angewiesen und müssen sich deshalb auch nach den Wünschen der Investoren richten, in der Erwartung, mit dem Zufluß von langfristigem ausländischem Kapital den kurzfristigen Abfluß von Gewinnen kompensieren zu können. Daher finden die Entwicklungsländer sich bereit, in allen Investitionsschutzverträgen den freien Gewinntransfer zuzusagen, sofern die aus ihrer Sicht unabdingbaren Voraussetzungen erfüllt sind: Alle Steuern müssen bezahlt sein, die ausgeschütteten Gewinne dürfen nicht den Liquiditätsstatus und das Wachstum des Unternehmens gefährden. Die Gewinne dürfen nur auf dem gesetzlich vorgeschriebenen Weg transferiert und

\footnotetext{
10 U. Amman, Der Schutz ausländischer Privatinvestitionen in Entwicklungsländern aus völkerrechtlicher, volkswirtschaftlicher und betriebswirtschaftlicher Sicht, Zürich/St. Gallen 1967, S. 145.

$11 \mathrm{Vgl}$. H. J. Abs, Die rechtliche Problematik privater Auslandsinvestitionen, Karlsruhe 1969, S. 112.

12 U. Amman, a. a. O., S. 26.

13 Ebenda, S. $57 \mathrm{ff}$.

$14 \mathrm{Vgl}$. B. Higgins, Economic Development. Principles, Problems and Policies rev. edition. London 1968, S. $562 \mathrm{f}$.

15 Vgl. z. B. P. O. Proehl, Foreign Enterprise in Nigeria. Laws and Policies, Chapel Hill 1965, S. 164.

16 Vgl. V. Tanzi, J. Aschheim, Saving Investment and Taxation in Underdeveloped Countries, in: Kyklos, Bd. 18, 1965, S. 222.
} 
müssen dafür bei der Notenbank des einzelnen Landes angemeldet werden ${ }^{17}$. Die ausländischen Eigentümer sollen für illegale Devisenexporte ihre Vorrechte wie den freien und unbeschränkten Gewinntransfer nicht mißbrauchen. Die Ausschüttungspolitik soll nicht die Leistungsfähigkeit der Unternehmen im Entwicklungsland beeinträchtigen.

Auf diese Weise stimmen Vereinbarungen in den Investitionsförderungsverträgen die unterschiedlichen wirtschaftlichen Standpunkte aufeinander ab. In den seltensten Fällen wird es aber eine deckungsgleiche Übereinstimmung geben, da die Ziele und Möglichkeiten der Entwicklungsländer und Industrieländer sich in vielen Punkten unterscheiden.

\section{Die Beziehung der Kapitalschutzabkommen zum nationalen Recht}

Probleme können sich jedoch daraus ergeben, daß Bestimmungen des Völkerrechts Ersatzansprüche der Bürger im Entwicklungsland präjudizieren. Denn im Gegensatz $\mathrm{zu}$ den vergleichsweise seltenen Enteignungen ausländischer Unternehmen in Entwicklungsländern werden viele Bürger oder einzelne Gruppen (wie z. B. Großgrundbesitzer) enteignet, ohne daß sie besondere Ersatzansprüche geltend machen können. Aus den Forderungen, die nach den Kapitalschutzabkommen Ausländern bei Enteignungen entstehen, können wegen des Grundsatzes der Gleichbehandlung von In- und Ausländern Ansprüche erhoben werden, die bisher versagt wurden. So sind zum Beispiel in den meisten Entwicklungsländern im Zuge der Landreform Großgrundbesitzer entschädigungslos oder nur zum Teil gegen geringe Ersatzleistungen enteignet worden. Die Regierungen in vielen Entwicklungsländern finden sich aber nicht bereit, aus finanziellen Erwägungen und im Hinblick auf die bestehenden gesellschaftspolitischen Ziele für diese Umverteilungsmaßnahmen Ersatz zu leisten. Denn die Enteignung soll u. a. die Umverteilung des Vermögens und vor allem des Grundbesitzes und der produktiveren Verwendung des Bodens herbeiführen. Eine hohe Ersatzleistung würde dieser angestrebten Wirkung zuwiderlaufen. Deshalb wird in den meisten Kapitalschutzabkommen die Ersatzleistungspflicht äußerst unklar formuliert. Indien verweigert sogar die Unterschrift bei Abkommen, die Ersatzleistungen vorsehen, da die Regierung die Ersatzansprüche der Maharadschas befürchtet ${ }^{18}$.

\section{Doppelbesteuerungsabkommen}

Wie die Investitionsförderungsverträge zwischen den nationalstaatlichen Eigentumsvorstellungen ausgleichen, sollen die Doppelbesteuerungsabkommen die Steuergesetze und Steuertarife in den Abkommensländern miteinander abstimmen. Dabei geht es nicht nur um die Angleichung der Steuergesetze im formalen und materiellen Sinn. Die Abkommen sollen vor allem die doppelte Besteuerung von Einkünften vermeiden, „die auf dem Wege von der Quelle bis zum Empfänger eine staatliche Steuergrenze überschreiten"19. Beispielsweise können Einkünfte bei

\footnotetext{
$17 \mathrm{Vgl}$. dazu die Ubersichten in den Annual Reports Exchange Restrictions des Internationalen Währungsfonds (IMF), Washington. o. J. (fortlaufende Jahrgänge).

$18 \mathrm{Vgl}$. H. J. Abs, a. a. O., S. 13.

19 G. Sass, Besteuerung nach den Doppelbesteuerungsabkommen. Von Dividenden und Zinsen, Frankfurt/M., 1968 , S. 11.
} 
der Entstehung und beim Empfänger zweimal besteuert werden. Die doppelte Belastung mindert den Ertrag des Investors und hemmt damit wegen der zwangsläufig geringeren Rendite auch den Kapitalexport in die Entwicklungsländer. Deshalb tun Industrie- und Entwicklungsländer alles, um die Doppelbesteuerung zu vermeiden. Dabei wird in zweiseitigen Verträgen eine Vereinbarung getroffen, die die Steuerquellen - das sind in der Regel bestimmte Einkünfte (Dividenden, Zinsen, Lizenzgebühren u. ä., Betriebsstätteneinkünfte) - dem einen oder anderen Land zuweisen. Ein Partnerland verzichtet auf diese Steuerquellen, indem es sie von der Besteuerung freistellt (Freistellungsmethode). Die deutschen Doppelbesteuerungsabkommen mit Entwicklungsländern stellen in der Regel die Betriebsstätteneinkünfte von der Besteuerung im Land des Empfängers frei. Dividenden, Zinsen und Lizenzgebühren werden im Industrie- und Entwicklungsland besteuert. Um die Doppelbesteuerung zu vermeiden, vereinbaren die das Abkommen schließenden Länder deshalb, die Steuern des Landes, in dem die Einkünfte entstanden sind, in dem Land des Wohnsitzes des Empfängers zu einem bestimmten Teil anzurechnen (Anrechnungsmethode) ${ }^{20}$. Die Vorteile der einzelnen Methoden sind in der Vergangenheit Gegenstand langwieriger Auseinandersetzungen gewesen. Sie geben noch heute den Stoff für internationale Steuerfachkonferenzen $a b$, ohne daß neue Ergebnisse erarbeitet werden ${ }^{21}$. Dabei wird übersehen, daß beide Wege zur Vermeidung der Doppelbesteuerung die tatsächliche Doppelbesteuerung nicht ausschließen müssen. So setzt die Anrechnungsmethode voraus, daß die Steuersätze in beiden Ländern gleich hoch und unbeschränkt anrechenbar sind. Höhere Steuern für deutsche Investoren in dem Entwicklungsland, die nicht in vollem Umfang auf die deutsche Steuerschuld angerechnet werden, führen wirtschaftlich schon zu einer Doppelbesteuerung. Dies kann auch nicht die Freistellungsmethode unterbinden. Beispielsweise können im Industrieland Ergänzungssteuern auf Einkünfte erhoben werden, die den Abkommen nach nur im Ausland besteuert werden dürfen.

Darüber hinaus können weitere Maßnahmen der Industrieländer zugunsten der Entwicklungsländer die tatsächliche Doppelbesteuerung trotz entgegengesetzter Vereinbarungen hervorrufen. Um zum Beispiel den Gewinntransfer aus Entwicklungsländern $\mathrm{zu}$ begrenzen und die steuerlich bedingte Abwanderung von Einkünften in Entwicklungsländer mit niedrigen Steuern zu unterbinden, stellten die amerikanischen Steuerbehörden Gewinne aus bestimmten Entwicklungsländern von der Steuer frei, erhoben aber eine eigene Steuer, wenn die Gewinne transferiert wurden (Western Hemisphere Trade Company) ${ }^{22}$.

\section{Nationales Steuerrecht als Ursache von Benachteiligungen}

In der BRD ist dieser Vorbehalt gegen die Maßnahmen zur Vermeidung der Doppelbesteuerung nicht gerechtfertigt. Allerdings führte die folgerichtige Anwendung der Freistellungsmethode zu „strukturellen Nachteilen“, die zum Teil erst

20 Vgl. O. Bühler, Prinzipien des Internationalen Steuerrechts, München/Berlin 1965, S. $193 \mathrm{ff}$.

21 Vgl. z. B. Beschluß auf dem XVI. IFA (International Fiscal Association)-Kongreß in Jerusalem 1961, in: IFA-Vorträge, Köln 1967, S. $39 \mathrm{ff}$; ; UN, Tax Treaties between Developed and Developing Countries, New York 1969, S. $18 \mathrm{ff} .$, S. $41 \mathrm{ff}$.

22 Vgl. C. Bellstedt, Die Steuer als Instrument der Politik. Eine vergleichende Untersuchung der Steuerpolitik in den USA und Deutschland, Berlin 1966, S. 387, G. Grasmann, Steuerliche Maßnahmen zur För derung der privaten Direktinvestitionen in Entwicklungsländern, in: Außenwirtschaftsdienst des BetriebsBeraters, 6. Jg. 1960, S. 259. 
im Steueränderungsgesetz 1969 beseitigt wurden ${ }^{23}$. Denn die Freistellung hebt die im deutschen Steuerrecht üblicherweise angenommene enge Beziehung $z$ wischen der Betriebsstätte und dem zentralen Unternehmen auf. Die „künstliche Einheit“ zwischen der ausländischen Betriebsstätte und dem deutschen Unternehmen wird mit den Unterschieden in der Rechtsordnung des kapitalgebenden und des kapitalnehmenden Landes aufgelöst. Deutsche Unternehmen durften deshalb die Verluste der Betriebsstätten nicht gegen die in der BRD ermittelten Gewinne aufrechnen. Wenn der deutsche Fiskus schon nicht von den Einkünften deutscher Betriebsstätten im Ausland Steuern erheben konnte, dann wollte er sich auch nicht noch mit Steuer-Aufkommensminderungen an den Verlusten im Ausland beteiligen. Zusätzlich wurden technisch-administrative Bedenken gegen die Verlustanrechnung geltend gemacht. Die notwendige Änderung der bis dahin geltenden Bestimmungen brachte erst das Steueränderungsgesetz 1969.

Ebenso wurde das in den Doppelbesteuerungsabkommen enthaltene Schachtelprivileg auf die Länder ausgedehnt, mit denen bisher noch keine Abkommen geschlossen worden waren. Der Verlustübertrag über die Grenzen wurde gleichzeitig den inländischen Regelungen für in der BRD gelegene Tochterunternehmen gleichgestellt ${ }^{24}$. Lediglich mit 9 Entwicklungsländern bestehen Doppelbesteuerungsabkommen für die Einkommen- und Körperschaftssteuern, das sind weniger als $10 \%$ aller Entwicklungsländer (Vgl. Tabelle 3). Auf sie entfielen bis zum Sommer 1970 754,5 Mio. DM an deutschen Direktinvestitionen, 13,1\% aller deutschen Direktinvestitionen in Entwicklungsländern ${ }^{25}$.

Mit der Gleichstellung aller Länder gegenüber dem deutschen Steuerrecht gewinnen auch andere Länder als Standorte für deutsche Investoren an Bedeutung, mit denen bisher noch kein Abkommen geschlossen worden war und/oder mit denen auch keines geschlossen werden soll. Denn der Aufwand für solche Abkommen verpflichtet zur Auswahl der Partnerländer. Die einzelnen Abkommen werden darüber hinaus erst nach mehrjährigen Verhandlungen ratifiziert und versprechen daher nur langfristig Nutzen.

\section{Tabelle 3}

\section{Deutsche Doppelbesteuerungsabkommen und Kapitalschutzabkommen} mit Industrie- und Entwicklungsländern im internationalen Vergleich

\begin{tabular}{|c|c|c|c|c|c|}
\hline & & & $\begin{array}{r}\text { Doppelbe } \\
\text { abko }\end{array}$ & $\begin{array}{l}\text { erungs- } \\
\text { en }^{1}\end{array}$ & $\begin{array}{c}\text { Kapitalschutz- } \\
\text { abkommen }{ }^{2}\end{array}$ \\
\hline & & & $\begin{array}{c}\text { mit Entwicklungs- } \\
\text { ländern }\end{array}$ & $\begin{array}{l}\text { mit Industrie- } \\
\text { ländern }\end{array}$ & $\begin{array}{c}\text { mit Entwicklungs- } \\
\text { ländern }\end{array}$ \\
\hline BRD & . & . . & . . 9 & 17 & 38 \\
\hline USA & . . & . . & . . 9 & 22 & 11 \\
\hline Schweiz & $\cdot$ & . . & . . . - & 13 & 19 \\
\hline $\begin{array}{l}\text { Großbri } \\
\text { (United }\end{array}$ & & $\begin{array}{l}\text { ien } \\
\text { gdom) }\end{array}$ & . . . 41 & 21 & 1 \\
\hline
\end{tabular}

$23 \mathrm{Vgl}$. P. Swoboda, Besteuerung und internationale wirtschaftliche Beziehungen, in: Zeitschrift für betriebswirtschaftliche Forschung, 21. Jg., N. F. 1969, S. 192; o. V. Sofortprogramm zur Förderung der Direktinvestitionen im Ausland. Vorschläge für neue Maßnahmen zur langfristigen Sicherung des außenwirtschaftlichen Gleichgewichts, in: Bulletin des Presse- und Informationsamtes der Bundesregierung, Nr. 374, 9. 4. 1969, S. $374 / 375$

24 Vgl. H. Vogel, Zur steuerlichen Belastung zwischenstaatlicher Kapitalinvestitionen unter besonderer Berücksichtigung des Außensteuerrechts der Bundesrepublik Deutschland, Düsseldorf 1964, S. 105.

25 Berechnet nach Angaben des Bundesministeriums der Wirtschaft. 
Anmerkung:

1 Nur Abkommen, die die Einkommens- und Vermögensbesteuerung betreffen.

2 Kapitalschutzabkommen werden nur mit Entwicklungsländern abgeschlossen.

Quelle:

UN, The Role of Private Enterprise in Investment and Promotion of Exports in Developing Countries, Report by D. U. Stikker, New York, 1968, S. 24, Fußn. 56, o. V. IHK: Höherer Investitionsschutz durch bilaterale Verträge mit den Entwicklungsländern, in: Nachrichten für Außenhandel, Nr. 296 vom 22. Dezember 1970; UN, International Tax Agreements, New York, Stand 1968.

\section{Nationale Regelungen unausweichlich}

Die Zahl bilateraler Abkommen mit Entwicklungsländern ist gering. Der Schutz reicht schon deshalb nicht aus. Darüber hinaus sichern die Bestimmungen in den Vereinbarungen die Investoren nur gegen Enteignungen und ähnliche Eingriffe sowie gegen die doppelte Besteuerung. Doch das sind nur einige Probleme bei der Vornahme von Direktinvestitionen in Entwicklungsländern. Deshalb muß das System zwischenstaatlicher Vereinbarungen zur Förderung von langfristigen Kapitalanlagen in der Dritten Welt durch nationale Regelungen ergänzt werden, die Verluste bei Enteignungen ersetzen und/oder mit steuerlichen Erleichterungen Finanzierungshilfen geben ${ }^{26}$. Die Beteiligung staatlicher Gesellschaften aus den Industrie- und/oder den Entwicklungsländern soll durch Risikovertreibung die Furcht vor Enteignungen oder anderen Behinderungen im Gastland herabsetzen $^{27}$.

So wurde 1962 die Deutsche Gesellschaft für wirtschaftliche Zusammenarbeit (Entwicklungsgesellschaft) $\mathrm{mbH}$ (DEG) gegründet. Sie „tritt bei Investitionsvorhaben in Entwicklungsländern als Vermittler und Bankier auf, indem sie Beteiligungen am haftenden Kapital übernimmt oder beteiligungsähnliche Darlehen gewährt". Dabei "arbeitet die DEG eng mit den Hausbanken der Unternehmer zusammen". Die Haftung ist auf einen festen Betrag begrenzt und an die üblichen Mitspracheund Kontrollrechte sowie an eine Gewinnbeteiligung gebunden ${ }^{28}$. Für Verluste bei Kapitalanlagen infolge politischer Ereignisse (wie Verstaatlichung, Enteignung, enteignungsgleicher Eingriffe, Kriegsfall, Revolution oder Aufruhr, Zahlungsverbote oder Moratorien, Unmöglichkeit der Konvertierbarkeit oder des Transfers) übernimmt die Bundesrepublik Deutschland eine Haftung für 15 bis 20 Jahre. Zurückgezahlt werden höchstens $95 \%$ (bis 1970 90\%) des Ablagewertes. Neben einer Selbstbeteiligung von $5 \%$ (früher 10\%) müssen jährlich 0,5\% des Auslandsvermögens als laufendes Entgelt entrichtet werden. Daneben wird eine Bearbeitungsgebühr zwischen 0,5 und $1 \%$ je nach Höhe der Garantiesumme erhoben ${ }^{29}$.

Ergänzt werden diese Förderungsmaßnahmen für Direktinvestitionen in Entwicklungsländern durch Finanzierungszuschüsse aus dem ERP-Sondervermögen und mit Darlehen aus dem Bundeshaushalt nach dem Allgemeinen Kriegsfolgegesetz ( $\mathbb{8} 85)$.

$26 \mathrm{Vgl}$. H. J. Abs, a. a. O., S. 6.

$27 \mathrm{Vgl}$. OECD, Investing in Developing Countries, Facilities for the Promotion of Foreign Private Investment in Developing Countries, Paris 1970; UN, The Role of Private Enterprise in Investment and Promotion of Exports in Developing Countries. Report by D. U. Stikker, New York 1968; Institut Finanzen und Steuern e. V. Probleme der Entwicklungshilfe, a. a. O., o. J., S. $33 \mathrm{ff}$.

28 Vgl. D. Kebschull, Direktinvestitionen im Ausland. Ansatzpunkte und Förderungsinstrumente, in: Wirtschaftsdienst, 49. Jg. 1969, S. 484, sowie die jährlichen Geschäftsberichte der DEG.

29 Vgl. ebenda, S. 483 und ÓECD, Investing in Developing Countries, a. a. O., S. $50 \mathrm{ff}$. 
Dazu kommen indirekte Finanzhilfen nach dem Entwicklungshilfesteuergesetz, das die Bildung einer Rücklage für 6 Jahre vorsieht für die Vornahme der Direktinvestitionen. Sie muß steuererhöhend nach 6 Jahren mit je $1 / 6$ innerhalb weiterer 6 Jahre aufgelöst werden. Daneben erlaubt das Entwicklungshilfesteuergesetz einen steuerfreien Abschlag von $15 \%$. Im Anlagejahr erhält der Investor damit einen Steuerkredit von $37 \%$, die gesamte Steuerersparnis beläuft sich je nach Berechnungsweise auf etwa $18 \% 0^{30}$.

Diese rein nationalen Maßnahmen heben sich im internationalen Vergleich von dem Förderungsinstrumentarium anderer Länder $a b$. Nach einer Übersicht der OECD hat damit die Bundesrepublik Deutschland trotz aller Unvollkommenheiten eines der besten und wirksamsten Systeme, um langfristige Kapitalanlagen in Entwicklungsländern den deutschen Unternehmen nahezulegen - ein Ausdruck für das Bemühen der BRD um die private Entwicklungshilfe mit Direktinvestitionen und die Schwierigkeiten, vor denen die deutschen Investoren stehen ${ }^{31}$.

30 Vgl. K. W. Menck, Wieviel taugt das Entwicklungshilfe-Steuergesetz, in: Wirtschaftsdienst, 50. Jg. 1970, V. 143 ff. OECD, Investing in Developing Countries, a. 2. O., S. 49. 